\title{
The effect of atmospheric doping on pressure-dependent Raman scattering in supported graphene
}

\author{
Egor A. Kolesov ${ }^{1}$, Mikhail S. Tivanov ${ }^{* 1}{ }^{\S}$, Olga V. Korolik ${ }^{1}$, Olesya O. Kapitanova ${ }^{2}$, \\ Xiao $\mathrm{Fu}^{3}$, Hak Dong $\mathrm{Cho}^{3}$, Tae Won Kang ${ }^{3}$ and Gennady N Panin ${ }^{3,4}$
}

\author{
Full Research Paper \\ Address: \\ ${ }^{1}$ Belarusian State University, 4 Nezavisimosti Av., 220030 Minsk, \\ Belarus, ${ }^{2}$ Department of Chemistry, Moscow State University, \\ Leninskie Gory, 1, b.3, 119991, Moscow, Russia, ${ }^{3}$ Department of \\ Physics, Quantum-Functional Semiconductor Research Center, Nano \\ Information Technology Academy, Dongguk University, 3-26 Pildong, \\ Junggu, 100-715, Seoul, Korea and ${ }^{4}$ Institute for Microelectronics \\ Technology \& High Purity Materials, RAS, 142432 Chernogolovka, \\ Moscow district, Russia \\ Email: \\ Mikhail S. Tivanov* - tivanov@bsu.by \\ * Corresponding author \\ § Phone +375172095451; Fax +375172095445 \\ Keywords: \\ adsorption; doping; graphene; pressure; Raman spectroscopy; \\ substrate \\ Beilstein J. Nanotechnol. 2018, 9, 704-710. \\ doi:10.3762/bjnano.9.65 \\ Received: 04 October 2017 \\ Accepted: 23 January 2018 \\ Published: 22 February 2018 \\ Associate Editor: A. J. Meixner \\ (ㄷ) 2018 Kolesov et al.; licensee Beilstein-Institut. \\ License and terms: see end of document.
}

\begin{abstract}
Atmospheric doping of supported graphene was investigated by Raman scattering under different pressures. Various Raman spectra parameters were found to depend on the pressure and the substrate material. The results are interpreted in terms of atmospheric adsorption leading to a change in graphene charge carrier density and the effect of the substrate on the electronic and phonon properties of graphene. It was found that adsorption of molecules from the atmosphere onto graphene doped with nitrogen (electron doping) compensates for the electron charge. Furthermore, the atmosphere-induced doping drastically decreases the spatial heterogeneity of charge carriers in graphene doped with nitrogen, while the opposite effect was observed for undoped samples. The results of this study should be taken into account for the development of sensors and nanoelectronic devices based on graphene.
\end{abstract}

\section{Introduction}

Graphene is a promising material for a variety of applications due to its unique physical properties [1]. Among its other outstanding features, one can distinguish its strong sensitivity to adsorbates, leading to a huge number of potential graphene applications in biosensors [2]. Atmospheric adsorption is known to affect graphene charge carrier density, leading to gradual self-sustained hole doping [3]. On one hand, adsorption can thus be an extremely undesirable effect when a nanoelectronic device is designed to operate on the basis of undoped properties of graphene; on the other hand, the adsorption of certain functional groups, for example, can be used as a constituent part of the methods of graphene functionalization [4]. At the same 
time, graphene layers on substrates are necessary for nanoelectronics. The substrates may affect the adsorption of graphene, either enhancing it, an effect very useful for biosensor applications [2], or reducing it, which is desirable when undoped graphene is needed as a device functional element.

A natural way for probing the adsorption properties of graphene is to conduct experiments under a low-pressure environment Various high-pressure measurements for graphene are presented in the literature $[5,6]$, while to the best of our knowledge, the studies of graphene's response to pressure variations below atmospheric values are absent to date. As the pressure decreases, both graphene and the substrate will expand, and thus, the expected changes in the properties of graphene will not be only related to graphene's compressibility, but also to strain caused by different compressibility values for graphene and the substrate. The authors of [5] have provided a convenient expression for compressibility-induced changes, while strain-related changes can be treated in a similar way to the thermal expansion description for supported graphene [7,8], where the strain is caused by a difference of thermal expansion coefficients for graphene and the substrate. Besides, monomers and functional groups adsorbed from the atmosphere are expected to become at least partially removed from the graphene surface as the pressure decreases, leading to changes in atmosphere-induced doping of the material [3]. A study of the characteristics of these processes may provide useful information about the adsorption properties of supported graphene.

Raman spectroscopy is a versatile tool for studying graphene and graphene-based nanomaterials [9-12]. Several different parameters of graphene's Raman spectra are affected by charge carrier density variations, such as the $\mathrm{G}$ and $2 \mathrm{D}$ peak positions and the ratio of the $2 \mathrm{D}$ and $\mathrm{G}$ peak maximum intensities [13]. Since the sensitivity of these parameters to doping takes place due to fundamentally different processes, using a set of these parameters provides the possibility to accurately determine charge carrier density variations and distinguish the latter against, for example, substrate-induced strain effects $[14,15]$.

The purpose of this study is to investigate the pressure-dependent behavior of the density of charge carriers in graphene and the effect of the substrate on the adsorption properties of graphene at pressures below atmospheric pressure.

\section{Results and Discussion Pressure-dependent Raman measurements}

Raman spectra of graphene on various substrates under atmospheric pressure are presented in Figure 1. Monolayer and bilayer graphene were identified by 2D peak approximations with one and four Lorentz functions, respectively, together with the
$2 \mathrm{D}$ peak full-width at half-maximums (FWHMs). The $I_{2 \mathrm{D}} / I_{\mathrm{G}}$ intensity ratio shows values of up to 1.5 for the monolayer and about 0.9 for bilayer graphene (the low $I_{2 \mathrm{D}} / I_{\mathrm{G}}$ values can be explained by adsorption doping [9] and electrostatic doping effects [16], as analyzed throughout the article).

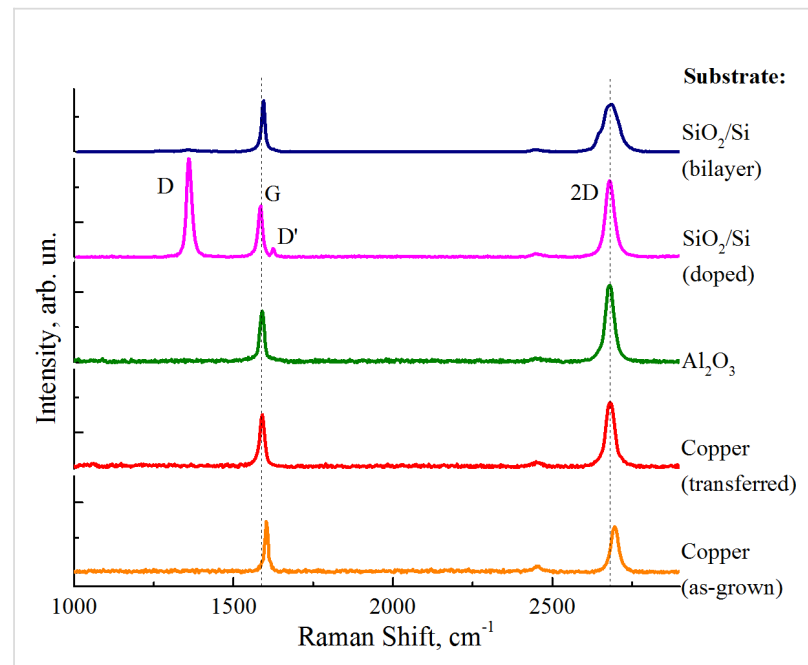

Figure 1: Raman spectra of graphene on various substrates, as well as bilayer and nitrogen-doped graphene.

Comparing the spectra for as-grown and transferred graphene on copper, one can distinguish the upshifted positions of the $G$ and $2 \mathrm{D}$ peaks. The stronger upshift for as-grown graphene (by $20-23 \mathrm{~cm}^{-1}$ with respect to normal positions) can be attributed to several effects: substrate-induced strain $[14,15]$, hole doping by adsorbates [13], and substrate-induced electrostatic doping [16]. The $G$ and $2 D$ peak positions upshifted by $5-8 \mathrm{~cm}^{-1}$ and the considerably low $I_{2 \mathrm{D}} / I_{\mathrm{G}}$ ratio of $1.3-1.4$ for graphene transferred onto copper and $\mathrm{Al}_{2} \mathrm{O}_{3}$ could be explained by similar effects, which in this case, tend to be less pronounced after the transfer. For doped graphene $(1.11 \%$ of nitrogen from XPS data), the D band intensity increases compared to pristine graphene, the second disorder-induced peak (the D' band) also becomes evident, the $\mathrm{G}$ peak position is upshifted by $\approx 3 \mathrm{~cm}^{-1}$, while the $2 \mathrm{D}$ peak is about normal, naturally indicating electron doping together with $I_{2 \mathrm{D}} / I_{\mathrm{G}}$ values of 1.4-1.6 [13].

Figure 2 shows pressure-dependent $\mathrm{G}$ and 2D peak shifts, typical for different samples, with respect to normal positions of about 1582 and $2676 \mathrm{~cm}^{-1}$ for a $532 \mathrm{~nm}$ laser, respectively. Downshift was commonly observed for undoped graphene peaks as the pressure decreased, while for nitrogen-doped graphene, the $\mathrm{G}$ peak position value increased. The $2 \mathrm{D}$ peak remained unchanged throughout the whole pressure range. Besides, changes of the $I_{2 \mathrm{D}} / I_{\mathrm{G}}$ intensity ratio were observed as the pressure decreased, indicating variations of graphene charge 

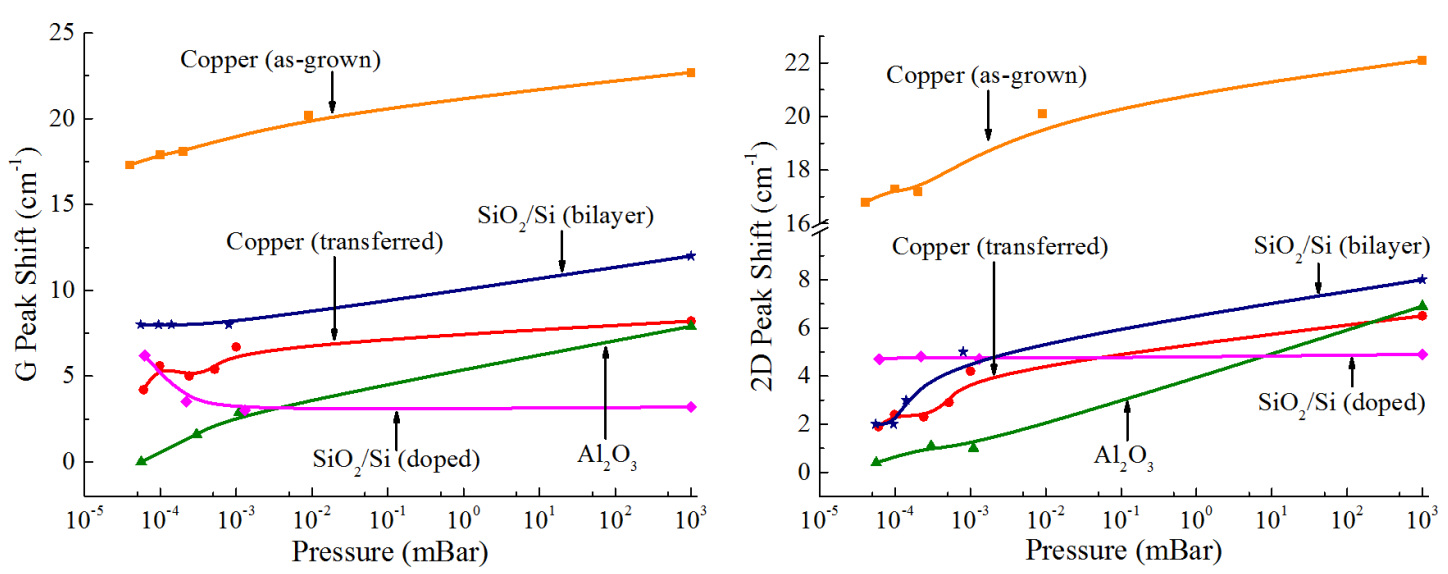

Figure 2: Pressure-dependent G and 2D peak position shifts for graphene on various substrates, as well as bilayer and nitrogen-doped graphene. Symbols show the experimental points, and solid lines represent general trends of peak shift.

carrier density [13]. After the atmosphere was returned to that of the pressure-controlled box, the spectra parameters gradually changed back to their initial values over a period of up to four days of air exposure.

\section{Theoretical estimation of different process contributions to the observed shift}

The $\mathrm{G}$ and $2 \mathrm{D}$ peak experimental pressure shift $\Delta \omega_{\mathrm{G}, 2 \mathrm{D}}^{\exp }$ can be explained by changes in the corresponding phonon mode energy due to (1) graphene lattice compressibility $\Delta \omega_{\mathrm{G}, 2 \mathrm{D}}^{\text {latt }}$ [5], (2) strain induced by the difference of compressibility values for graphene and substrate $\Delta \omega_{\mathrm{G}, 2 \mathrm{D}}^{\varepsilon}[5,14]$, and (3) possible desorption of atmosphere-induced doping groups $\Delta \omega_{\mathrm{G}, 2 \mathrm{D}}^{\text {doping }}[3,13]$ Thus, the experimental peak shift due to pressure change can be written as:

$$
\Delta \omega_{\mathrm{G}, 2 \mathrm{D}}^{\exp }=\Delta \omega_{\mathrm{G}, 2 \mathrm{D}}^{\mathrm{latt}}+\Delta \omega_{\mathrm{G}, 2 \mathrm{D}}^{\varepsilon}+\Delta \omega_{\mathrm{G}, 2 \mathrm{D}}^{\text {doping }} .
$$

The first term is defined by the following expression [5]:

$$
\Delta \omega_{\mathrm{G}, 2 \mathrm{D}}^{\mathrm{latt}}=\omega_{\mathrm{G}, 2 \mathrm{D}}^{0}\left[\frac{a(p)}{a_{0}}\right]^{-2 \gamma_{\mathrm{G}, 2 \mathrm{D}}}-\omega_{\mathrm{G}, 2 \mathrm{D}}^{0},
$$

where $\omega_{\mathrm{G}}^{0}$ and $\omega_{2 \mathrm{D}}^{0}$ are normal $\mathrm{G}$ and 2D peak positions, respectively; $\gamma_{\mathrm{G}, 2 \mathrm{D}}$ is a Grüneisen parameter for corresponding $\mathrm{E}_{2 \mathrm{~g}}$ and $\mathrm{A}_{1 \mathrm{~g}}$ phonon modes (values of 1.8 and 2.7 were used, respectively [14]), $a$ is the graphene atomic bond length ( $a_{0}$ under zero-pressure conditions). As it was shown in [5], a relationship for graphite in-plane compressibility from [17] is applicable for $a(p) / a_{0}$ estimation:

$$
\frac{a(p)}{a_{0}}=\left[p \frac{\beta^{\prime}}{\beta_{0}}+1\right]^{-1 / \beta^{\prime}}
$$

where

$$
\beta_{0}^{-1}=-\left.\frac{d \ln a}{d p}\right|_{p=0}
$$

is the linear compressibility and $\beta^{\prime}$ is its pressure derivative. In the present study, $\beta_{0}$ and $\beta^{\prime}$ values of $1250 \mathrm{GPa}$ and 1 were used, respectively [17].

The strain-induced shift, by analogy with thermal expansion strain $[7,8,18]$, can be written as:

$\Delta \omega_{\mathrm{G}, 2 \mathrm{D}}^{\varepsilon}=\frac{\partial \omega_{\mathrm{G}, 2 \mathrm{D}}}{\partial \varepsilon}\left[\left(\frac{r(p)}{r_{0}}\right)_{\text {substrate }}^{3}-\left(\frac{a(p)}{a_{0}}\right)_{\text {graphene }}^{2}\right]$

where

$$
\frac{\partial \omega_{\mathrm{G}, 2 \mathrm{D}}}{\partial \varepsilon}
$$

is a biaxial strain rate (in our calculations we used the values of -58 and $-144 \mathrm{~cm}^{-1} / \%$ for $\mathrm{G}$ and $2 \mathrm{D}$ peak, respectively [14]), $\varepsilon$ is a relative deformation (\%), and $r$ is a substrate atomic bond length.

In [5] it was shown that at low pressure the $r(p) / r_{0}$ relation can be approximately defined through the substrate bulk modulus $B_{0}$ in the following way: 


$$
\frac{d}{d p}\left[\frac{r(p)}{r_{0}}\right] \approx \frac{1}{3 B_{0}} .
$$

A value for the doping contribution $\Delta \omega_{\mathrm{G}, 2 \mathrm{D}}^{\text {doping }}$ can on one hand be calculated from Equation 1 using the obtained experimental shift values; on the other hand, in can be obtained from other doping-dependent Raman spectra parameters that are not commonly referred to as strain-dependent, such as $I_{2 \mathrm{D}} / I_{\mathrm{G}}$ intensity ratio [13].

During the calculation, it turned out that the graphene lattice compression contribution $\Delta \omega_{\mathrm{G}, 2 \mathrm{D}}^{\text {latt }}$ does not lead to a substantial change in the $\mathrm{G}$ and $2 \mathrm{D}$ peak positions - less than $10^{-2} \mathrm{~cm}^{-1}$ as the pressure changes from 0 to $1 \mathrm{~atm}\left(\approx 1 \cdot 10^{5} \mathrm{~Pa}\right)$. The same is valid for the strain-induced shift $\Delta \omega_{\mathrm{G}, 2 \mathrm{D}}^{\varepsilon}$ (less than $10^{-3} \mathrm{~cm}^{-1}$ for graphene on all substrates). Since these results dramatically exclude the first two terms from Equation 1, we assume the experimental shift to be caused by changes in graphene doping.

\section{Carrier density variation analysis}

Table 1 demonstrates the carrier density change $\Delta n$ obtained from Raman spectra [13] of various graphene samples as the pressure decreased, as well as Raman feature parameters at normal and reduced pressures. As can be seen, greater $\Delta n$ values were obtained for nitrogen-doped graphene, while for bilayers on $\mathrm{SiO}_{2} / \mathrm{Si}$ and graphene transferred to copper, weaker desorption occurred. It is important to note that doping has various effects on the Raman spectral parameters [13]: stronger doping is expressed as lower $I_{2 \mathrm{D}} / I_{\mathrm{G}}$ ratio values, while the $\mathrm{G}$ and 2D peak positions depend on carrier density non-linearly.

In order to analyze the obtained results, we first compare the peak position shift for pristine and transferred graphene on $\mathrm{Cu}$ in Figure 2, where one can notice a similar behavior up to a certain constant. A considerable similarity of the peak position downshift indicates analogous kinetics of adsorbate removal from graphene surface. However, a different intercept leads to a $13-15 \mathrm{~cm}^{-1}$ distance between the plots. This observation can be explained by substrate-induced strain in as-grown graphene (leading to greater overall peak positions [14]): The van der Waals graphene-substrate interaction model may not be fully applicable for this case due to the possibility of chemical bonds present between graphene and copper [19] which are terminated after the transfer. Besides, this may lead to different values of the average graphene-substrate distance resulting in deviation of the density of states (DOS) from a simple Dirac cone [16] and a consequent shift of the Dirac point energy, leading to different substrate-induced doping that affects all Raman features related to carrier density but most likely do not change with the pressure (smaller overall values of $I_{2 \mathrm{D}} / I_{\mathrm{G}}$ intensity ratio). This explains the similar shift but different intercept for pristine and transferred graphene on $\mathrm{Cu}$ in Figure 2.

Comparing graphene transferred to $\mathrm{Cu}$ and $\mathrm{Al}_{2} \mathrm{O}_{3}$ as typical conducting and dielectric materials, a stronger carrier density change was obtained for the latter. As the pressure decreases, removal of adsorbates from the surface of graphene on $\mathrm{Al}_{2} \mathrm{O}_{3}$ results in the Raman spectra parameters becoming close to

\begin{tabular}{|c|c|c|c|c|c|}
\hline Sample & Pressure, mbar & $\operatorname{Pos}(G), \mathrm{cm}^{-1}$ & $\operatorname{Pos}(2 \mathrm{D}), \mathrm{cm}^{-1}$ & $I_{2 \mathrm{D}} / I_{\mathrm{G}}$ & $\Delta n, \times 10^{13} \mathrm{~cm}^{-2}$ \\
\hline \multirow{2}{*}{$\begin{array}{l}\text { graphene on copper } \\
\text { (as-grown) }\end{array}$} & 1000 & 1605 & 2696 & 0.9 & \multirow[t]{2}{*}{$\approx 0.6-0.9$ (hole doping) } \\
\hline & $5 \times 10^{-5}$ & 1599 & 2691 & 1.2 & \\
\hline \multirow{2}{*}{$\begin{array}{l}\text { graphene on copper } \\
\text { (transferred) }\end{array}$} & 1000 & 1590 & 2681 & 1.3 & \multirow[t]{2}{*}{$\approx 0.3-0.5$ (hole doping) } \\
\hline & $5 \times 10^{-5}$ & 1586 & 2676 & 1.6 & \\
\hline \multirow[t]{2}{*}{ graphene on $\mathrm{Al}_{2} \mathrm{O}_{3}$} & 1000 & 1590 & 2681 & 1.4 & \multirow[t]{2}{*}{$\approx 0.5-0.8$ (hole doping) } \\
\hline & $5 \times 10^{-5}$ & $1582^{a}$ & $2674^{a}$ & $2.3^{a}$ & \\
\hline \multirow[t]{2}{*}{ doped graphene on $\mathrm{SiO}_{2} / \mathrm{Si}$} & 1000 & 1585 & 2679 & 1.5 & \multirow[t]{2}{*}{$\approx 0.7-1.0$ (electron doping) } \\
\hline & $5 \times 10^{-5}$ & 1588 & 2679 & 1.2 & \\
\hline \multirow[t]{2}{*}{ bilayer on $\mathrm{SiO}_{2} / \mathrm{Si}$} & 1000 & 1594 & 2682 & 0.9 & \multirow[t]{2}{*}{$\approx 0.3-0.5$ (hole doping) } \\
\hline & $5 \times 10^{-5}$ & 1590 & 2676 & 1.0 & \\
\hline
\end{tabular}

aThe closest to normal graphene peak positions as well as greater $I_{2 \mathrm{D}} / I_{\mathrm{G}}$ values were observed for graphene on $\mathrm{Al}_{2} \mathrm{O}_{3}$ at reduced pressure. 
those typical for completely undoped graphene [13] - in particular, the strong $I_{2 \mathrm{D}} / I_{\mathrm{G}}$ increase seen in Table 1 should be noted. At the same time, a smaller hole density decrease for graphene transferred to $\mathrm{Cu}$ can be explained by analogy with as-grown graphene by stronger graphene-substrate interaction in this case (interaction energy of $0.72 \mathrm{~J} / \mathrm{m}^{2}$ against $\approx 0.47 \mathrm{~J} / \mathrm{m}^{2}$ for graphene on $\mathrm{Al}_{2} \mathrm{O}_{3}$ substrate [20,21]) and therefore more pronounced substrate-induced doping [16]. Besides, the electrostatic screening of the electron-electron interaction for graphene on a metallic substrate $[22,23]$ can contribute. The screening effect results in renormalization of the Fermi velocity, Dirac point velocity, and overall distortion of the Dirac cone [24], leading to a possible charge carrier density increase [25].

In the case of bilayer graphene, the desorption process leads to a density decrease of about $0.4 \times 10^{13} \mathrm{~cm}^{-2}$ hole, which is close to the case of graphene transferred to $\mathrm{Cu}$. Being a van der Waals structure on the scale of adsorption effects (in terms of interlayer bonding), bilayer graphene is known to have a slightly greater energy barrier for the adsorption process [26]. However, adsorption in this case can also lead to at least partial layer decoupling that results in the presence of biaxial strain [27]. Besides, values of $I_{2 \mathrm{D}} / I_{\mathrm{G}}$ did not indicate a strong doping presence. Thus, the overall greater $\mathrm{G}$ and $2 \mathrm{D}$ peak position values most likely are strain-related rather than an indication of greater atmosphere-induced doping.

A very interesting situation is observed for nitrogen-doped graphene. The results of pressure-dependent studies presented in Figure 2 have shown a G peak upshift, while the 2D peak position remained unchanged. This behavior together with the change of $I_{2 \mathrm{D}} / I_{\mathrm{G}}$ corresponds to an increase of electron density, according to dependencies presented in [13]. This fact leads to an interesting conclusion that adsorption hole doping most likely counterbalances electron concentration in nitrogen-doped graphene. A greater change of the carrier density within the ex- perimental pressure range compared to undoped graphene indicates that under electronic doping more active adsorption occurs, balancing the "excess" electron density through opposite-sign charge carrier introduction and subsequent recombination, and moving the Fermi level position closer to a normal value.

As seen from Figure 3a, in addition to an upshift, the initially distorted $G$ peak at normal pressure experiences stronger profile distortion as the pressure decreases, with the most asymmetric profile corresponding to the lowest pressure. This fact indicates that as desorption occurs, inhomogeneity of the charge carrier distribution increases, approaching conditions typical for doped graphene [28]. Thus, atmospheric adsorption not only counterbalances the excess electron concentration, but also reduces the charge carrier inhomogeneity, with the whole system evolving closer to equilibrium.

It is important to note that the opposite situation was observed for undoped graphene: as the pressure decreased (with desorption leading to doping decrease), the G peak (in addition to the downshift) evolved from a distorted lineshape at normal pressure to an almost symmetric one in vacuum $\left(\approx 5 \times 10^{-5} \mathrm{mbar}\right)$, as seen in Figure $3 \mathrm{~b}$. This fact means that the effect of atmospheric adsorption that reduces charge carrier inhomogeneity in graphene is quite unique for the electron-doped graphene case, while the inverse process is naturally typical for the pure material, as it was previously noted [28].

The results presented above demonstrate that pressure-dependent Raman spectroscopy is a convenient tool for probing atmosphere-induced doping in graphene. Low-pressure behavior of adsorbates present on the graphene surface and concomitant charge carrier properties strongly depend on the substrate material, as well as graphene doping and number of layers. These results can be taken into account during the development of any
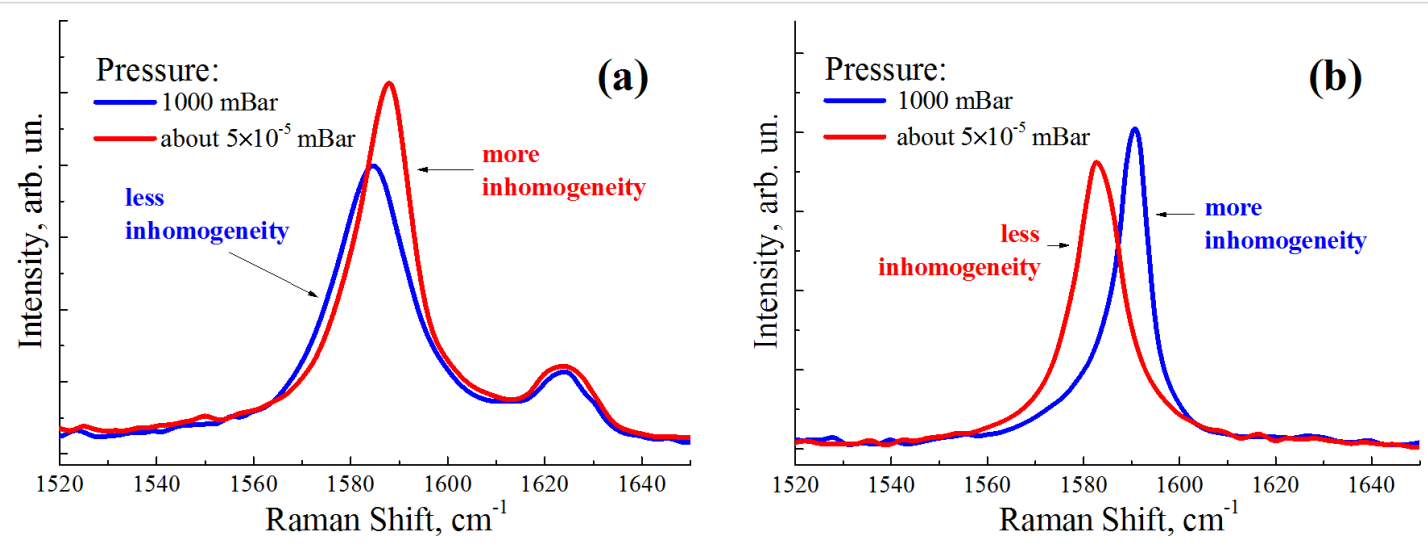

Figure 3: $\mathrm{G}$ peak profile for (a) nitrogen-doped graphene on $\mathrm{SiO}_{2} / \mathrm{Si}$ and (b) graphene on $\mathrm{Al}_{2} \mathrm{O}_{3}$ at normal and reduced pressures. 
graphene-related devices, either specifically involving graphene adsorption properties (for example, biosensors) or assuming exposure of air to the device functional elements.

\section{Conclusion}

Pressure-dependent Raman spectroscopy studies in the range from 1000 to $5 \times 10^{-5}$ mbar of supported graphene have shown that the $G$ and $2 D$ peak positions and the ratio of the $2 \mathrm{D}$ and $\mathrm{G}$ peak intensities change with the pressure, whereby the substrate material affects the dependencies. Performed calculations showed negligible lattice compressibility and strain contributions to the observed effect, and thus the shift was fully attributed to atmosphere-induced adsorption doping. During the analysis, the effect of the substrate on graphene electronic and phonon properties was taken into account. For nitrogen-doped graphene (electronic doping), adsorption was found to counterbalance the excess charge carriers. In addition, it turned out that atmosphere-induced doping decreases charge carrier inhomogeneity in nitrogen-doped graphene. For the undoped graphene samples, the opposite effect occurs. The results of the present study are useful for graphene-based sensor design, graphene functionalization techniques, and taking into account adsorption effects during nanoelectronic device engineering.

\section{Experimental}

Graphene was synthesized on $\mathrm{Cu}$ foil at $1020^{\circ} \mathrm{C}$ by a chemical vapor deposition (CVD) method using a mixture of $\mathrm{CH}_{4}$ of $40 \mathrm{sccm}$ and $\mathrm{H}_{2}$ of $10 \mathrm{sccm}$. Cu foil (Alfa Aesar, 99.999\%, $10 \times 30 \mathrm{~cm}^{2}, 25 \mu \mathrm{m}$ thick) was pre-annealed at $1060{ }^{\circ} \mathrm{C}$ with a hydrogen flow of $300 \mathrm{sccm}$ and an argon flow of 2,000 sccm at a pressure of $<10^{-4}$ Torr for 1-2 hours inside the chamber. After the growth of graphene, the $\mathrm{Cu}$ foil was cooled to room temperature. The cooling rate was described in detail elsewhere [29].

The grown graphene was transferred to various substrates using a "PMMA-mediated" method [30]. PMMA (molecular weight = 996000 , dissolved in anisole) was spin-coated (3000 rpm, $1 \mathrm{~min}$ ) on graphene supported by $\mathrm{Cu}$ foil. A $0.1 \mathrm{M}\left(\mathrm{NH}_{4}\right)_{2} \mathrm{~S}_{2} \mathrm{O}_{8}$ aqueous solution was used to etch $\mathrm{Cu}$ and a water/isopropyl alcohol mixture was used to wash graphene from etching products [31]. PMMA was removed by submerging the sample in glacial acetic acid (extra pure) [32] for $4 \mathrm{~h}$.

Graphene, doped with nitrogen, was prepared using a nitrogenplasma treatment described elsewhere [33].

The Raman spectra were obtained with a confocal Raman spectrometer Nanofinder HE (LOTIS TII) with a spectral resolution better than $3 \mathrm{~cm}^{-1}$. For the excitation of Raman radiation, a continuous wave solid-state laser with a wavelength of $532 \mathrm{~nm}$ was used. During low-pressure Raman measurements, the sample was studied in a pressure-controlled box (pressure ranged from 1000 to about $5 \times 10^{-5} \mathrm{mbar}$ ). A laser power of $2 \mathrm{~mW}$ was used, and the laser spot diameter was about $1.5 \mu \mathrm{m}$. Pressuredependent measurements were carried out in a typical graphene monolayer and a bilayer low-defect region.

\section{Acknowledgements}

This work was supported by Belarusian State Program for Research "Photonics, opto- and microelectronics" and Russian Foundation of Basic Researches (individual project 16-3360229), as well as partially supported by Basic Science Research Program through the National Research Foundation of Korea (NRF) funded by the Ministry of Education (ME) (No. 2017R1D1A1B03035102 and the International Research and Development program of NRF funded by the MEST (2017R1D1A1B03032759). The authors of this work are grateful to Ivan A. Svito for his help in organizing the experiment, and Prof. L.V. Yashina and Ph.D. Student A. I. Belova for XPS studies of nitrogen-doped graphene. This work was performed using equipment purchased within the Program of Development of the Lomonosov Moscow State University.

\section{ORCID ${ }^{\circledR}$ iDs}

Egor A. Kolesov - https://orcid.org/0000-0001-8917-8937

Mikhail S. Tivanov - https://orcid.org/0000-0002-4243-0813

Xiao Fu - https://orcid.org/0000-0002-0976-220X

Gennady N Panin - https://orcid.org/0000-0003-3658-6958

\section{References}

1. Zhu, Y.; Murali, S.; Cai, W.; Li, X.; Suk, J. W.; Potts, J. R.; Ruoff, R. S. Adv. Mater. 2010, 22, 3906-3924. doi:10.1002/adma.201001068

2. Shao, Y.; Wang, J.; Wu, H.; Liu, J.; Aksay, I. A.; Lin, Y. Electroanalysis 2010, 22, 1027-1036. doi:10.1002/elan.200900571

3. Ryu, S.; Liu, L.; Berciaud, S.; Yu, Y.-J.; Liu, H.; Kim, P.; Flynn, G. W.; Brus, L. E. Nano Lett. 2010, 10, 4944-4951. doi:10.1021/nl1029607

4. Chen, L.; Li, J.; Li, D.; Wei, M.; Wang, X. Solid State Commun. 2012, 152, 1985-1989. doi:10.1016/j.ssc.2012.04.054

5. Proctor, J. E.; Gregoryanz, E.; Novoselov, K. S.; Lotya, M.; Coleman, J. N.; Halsall, M. P. Phys. Rev. B 2009, 80, 073408. doi:10.1103/PhysRevB.80.073408

6. Filintoglou, K.; Papadopoulos, N.; Arvanitidis, J.; Christofilos, D.; Frank, O.; Kalbac, M.; Parthenios, J.; Kalosakas, G.; Galiotis, C.; Papagelis, K. Phys. Rev. B 2013, 88, 045418. doi:10.1103/PhysRevB.88.045418

7. Tivanov, M. S.; Kolesov, E. A.; Praneuski, A. G.; Korolik, O. V.; Saad, A. M.; Komissarov, I. V.; Kovalchuk, N. G. J. Mater. Sci.: Mater. Electron. 2016, 27, 8879-8883. doi:10.1007/s10854-016-4913-7

8. Kolesov, E. A.; Tivanov, M. S.; Korolik, O. V.; Saad, A. M.; Komissarov, I. V. Carbon 2017, 111, 587-591. doi:10.1016/j.carbon.2016.10.020

9. Ferrari, A. C.; Basko, D. M. Nat. Nanotechnol. 2013, 8, 235-246. doi:10.1038/nnano.2013.46 
10. Manikandan, E.; Kavitha, G.; Kennedy, J. Ceram. Int. 2014, 40, 16065-16070. doi:10.1016/j.ceramint.2014.07.129

11. Kaviyarasu, K.; Manikandan, E.; Kennedy, J.; Maaza, M. J. Mater. Sci.: Mater. Electron. 2016, 27, 13080-13085. doi:10.1007/s10854-016-5451-z

12. Kaviyarasu, K.; Maria Magdalane, C.; Manikandan, E.; Jayachandran, M.; Ladchumananandasivam, R.; Neelamani, S.; Maaza, M. Int. J. Nanosci. 2015, 14, 1550007. doi:10.1142/S0219581X15500076

13. Beams, R.; Cançado, L. G.; Novotny, L. J. Phys.: Condens. Matter 2015, 27, 083002. doi:10.1088/0953-8984/27/8/083002

14. Mohiuddin, T. M. G.; Lombardo, A.; Nair, R. R.; Bonetti, A.; Savini, G.; Jalil, R.; Bonini, N.; Basko, D. M.; Galiotis, C.; Marzari, N.; Novoselov, K. S.; Geim, A. K.; Ferrari, A. C. Phys. Rev. B 2009, 79, 205433. doi:10.1103/PhysRevB.79.205433

15. Frank, O.; Vejpravova, J.; Holy, V.; Kavan, L.; Kalbac, M. Carbon 2014, 68, 440-451. doi:10.1016/j.carbon.2013.11.020

16. Ulstrup, S.; Bianchi, M.; Hatch, R.; Guan, D.; Baraldi, A.; Alfè, D.; Hornekær, L.; Hofmann, P. Phys. Rev. B 2012, 86, 161402. doi:10.1103/PhysRevB.86.161402

17. Hanfland, M.; Beister, H.; Syassen, K. Phys. Rev. B 1989, 39, 12598. doi:10.1103/PhysRevB.39.12598

18. Yoon, D.; Son, Y.-W.; Cheong, H. Nano Lett. 2011, 11, 3227-3231. doi:10.1021/nl201488g

19. Das, S.; Lahiri, D.; Lee, D.-Y.; Agarwal, A.; Choi, W. Carbon 2013, 59, 121-129. doi:10.1016/j.carbon.2013.02.063

20. He, Y.; Chen, W. F.; Yu, W. B.; Ouyang, G.; Yang, G. W. Sci. Rep. 2013, 3, 2660. doi:10.1038/srep02660

21. Huang, B.; Xu, Q.; Wei, S.-H. Phys. Rev. B 2011, 84, 155406. doi:10.1103/PhysRevB.84.155406

22. Endlich, M.; Molina-Sánchez, A.; Wirtz, L.; Kröger, J. Phys. Rev. B 2013, 88, 205403. doi:10.1103/PhysRevB.88.205403

23. Siegel, D. A.; Hwang, C.; Fedorov, A. V.; Lanzara, A. New J. Phys. 2012, 14, 095006. doi:10.1088/1367-2630/14/9/095006

24. Siegel, D. A.; Regan, W.; Fedorov, A. V.; Zettl, A.; Lanzara, A. Phys. Rev. Lett. 2013, 110, 146802. doi:10.1103/PhysRevLett.110.146802

25. Li, J.; Tan, L. Z.; Zou, K.; Stabile, A. A.; Seiwell, D. J.; Watanabe, K.; Taniguchi, T.; Louie, S. G.; Zhu, J. Phys. Rev. B 2016, 94, 161406. doi:10.1103/PhysRevB.94.161406

26. Santos, H.; Henrard, L. J. Phys. Chem. C 2014, 118, 27074-27080. doi:10.1021/jp507178f

27. Chacón-Torres, J. C.; Wirtz, L.; Pichler, T. ACS Nano 2013, 7, 9249-9259. doi:10.1021/nn403885k

28. Casiraghi, C.; Pisana, S.; Novoselov, K. S.; Geim, A. K.; Ferrari, A. C. Appl. Phys. Lett. 2007, 91, 233108. doi:10.1063/1.2818692

29. Güneş, F.; Han, G. H.; Kim, K. K.; Kim, E. S.; Chae, S. J.; Park, M. H.; Jeong, H.-K.; Lim, S. C.; Lee, Y. H. Nano 2009, 4, 83. doi:10.1142/S1793292009001538

30. Liang, X.; Sperling, B. A.; Calizo, I.; Cheng, G.; Hacker, C. A.; Zhang, Q.; Obeng, Y.; Yan, K.; Peng, H.; Li, Q.; Zhu, X.; Yuan, H.; Hight Walker, A. R.; Liu, Z.; Peng, L.-m.; Richter, C. A. ACS Nano 2011, 5, 9144-9153. doi:10.1021/nn203377t

31. Gao, L.; Ni, G.-X.; Liu, Y.; Liu, B.; Castro Neto, A. H.; Loh, K. P. Nature 2014, 505, 190-194. doi:10.1038/nature12763

32. Her, M.; Beams, R.; Novotny, L. Phys. Lett. A 2013, 377, 1455-1458. doi:10.1016/j.physleta.2013.04.015

33. Wang, H.; Maiyalagan, T.; Wang, X. ACS Catal. 2012, 2, 781-794. doi:10.1021/cs200652y

\section{License and Terms}

This is an Open Access article under the terms of the Creative Commons Attribution License

(http://creativecommons.org/licenses/by/4.0), which permits unrestricted use, distribution, and reproduction in any medium, provided the original work is properly cited.

The license is subject to the Beilstein Journal of Nanotechnology terms and conditions: (https://www.beilstein-journals.org/bjnano)

The definitive version of this article is the electronic one which can be found at:

doi:10.3762/bjnano.9.65 